Part 3. Super Star Clusters and Associations Section A. Invited Reviews and Contributed Talks 


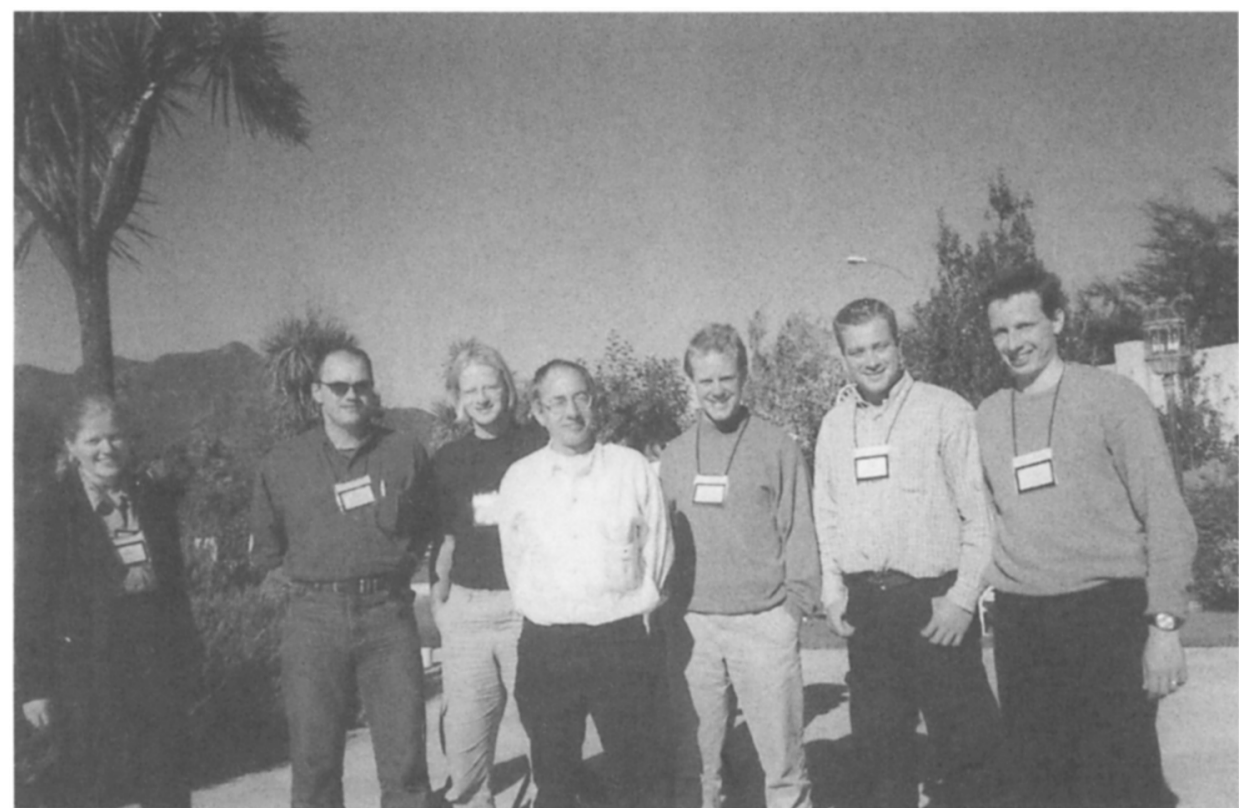

Herr Professor Tom Richtler (center) is surrounded by a Superstar cluster of his students: from left to right - Eva Grebel, Boris Dirsch, Michael Hilker, Markus Kissler-Patig, Matias Gomez and Soren Larsen. 


\title{
Super Stellar Clusters in HII Galaxies
}

\author{
Eduardo Telles \\ Observatório Nacional, Rua José Cristino, 77 \\ 20921-400 - Rio de Janeiro - Brasil
}

\begin{abstract}
Stellar Clusters are identified in images and in the spectra of these star forming dwarf galaxies. These Stellar Clusters have properties similar to those observed in other violent star forming galaxies and may the elementary entities of a starburst.
\end{abstract}

\section{Introduction}

Super Star Clusters (SSC) are found not only in the starburst regions of strongly interacting giant galaxies but also in dwarf galaxies in a bursting phase of star formation (SF), HII galaxies. The formation and evolution of these Stellar Clusters may be an important new ingredient in regulating the history of star formation in HII galaxies, including the triggering mechanism responsible for initiating this intense current episode of SF. Since their discovery in the early 70's, HII galaxies have gained the status of being the nearest galaxies with properties expected for truly young galaxies in the local universe. The question, posed by Sargent \& Searle (1970) of whether these are young galaxies forming stars for the first time seems now to have been answered by recent studies. H II galaxies show an underlying stellar population of intermediate to old age (Telles \& Terlevich 1997, Doublier et al. 1997, Marlowe et al. 1999, Cairós et al. 2001). Telles, Melnick \& Terlevich (1997) have classified a large sample of HII galaxies into two main groups: Type I includes the most luminous H II galaxies, all of which present an overall irregular morphology, and in many cases signs of being merger products. The low luminosity Type II's, on the other hand, are more compact and rounder showing no evidence of irregular isophotes or any sign of being disturbed by external agents. Type II HII galaxies are the ones which mostly challenge our understanding of how SF was initiated.

In this contribution I will review some of our own recent work, in which my collaborators and I have been involved, in the study of star formation in HII galaxies. It is not my intention to review other recent work in this area, but I point the reader to the review by Ostlin \& Kunth (2000).

\section{What Triggers the Starburst in HII Galaxies?}

Early studies of the environmental or clustering properties of HII galaxies have agreed that these are isolated galaxies with a deficit of $L>L^{*}$ galaxies within $1 \mathrm{Mpc}$ (Campos-Aguilar et al. 1993, Vilchez 1995, Telles \& Terlevich, 1995). These compelling studies have shown that HII galaxies are not associated with 


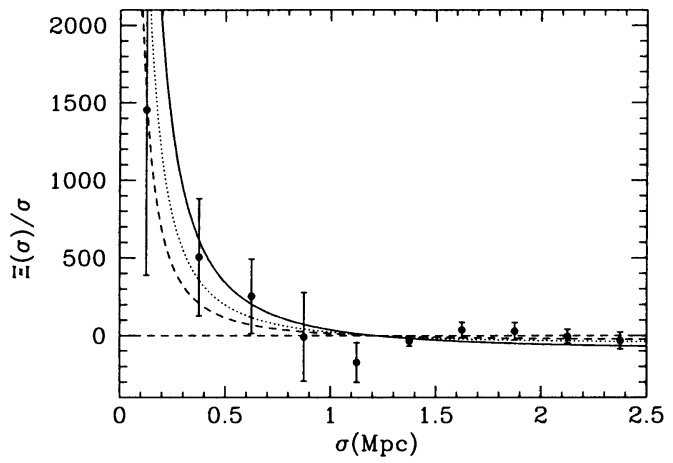

Figure 1. The projected cross-correlation between the HII galaxies and the faint APM field galaxies, $\Xi_{h g}(\sigma) / \sigma$. The points show our measurements. The solid, dotted and dashed lines show the predicted $\Xi$ for $r_{0}=5.1,3.7$ and $2.7 h^{-1}$ Mpc respectively. From Telles \& Maddox (2000).

giant galaxies, therefore they are not tidal debris of strongly interacting systems. These results show there is no evidence for external triggers being responsible for the onset of the current violent star formation episode.

An alternative was first proposed by Melnick (1987) that HII galaxies would be triggered not by giant galaxies but by other dwarfs or intergalactic HI clouds. Taylor and collaborators followed up these ideas and using the VLA detected 12 HI companions around $21 \mathrm{HII}$ galaxies, while only 4 HI-rich companions were detected around a control sample of 17 quiescent low surface brightness dwarfs (LSBD) (Taylor 1997 and references therein). Despite the effort by this group, these results were not able to, beyond reasonable doubt, settle the question of the possible effect of low mass companions as external triggers of the SF in HII galaxies, because some HII galaxies do not show HI companions while some LSBD do show HI companions and these are not 'bursting'!

Telles \& Maddox (2000) went further, in an attempt to supersede these small number statistics and put further constraints on the existence and/or properties of possible low mass companions. We carried out an investigation of the galaxy environments of a sample of over 160 low redshift HII galaxies by cross-correlating their accurate position in the sky to faint field galaxies in the APM galaxy catalog. By using the fact that all detectable HI clouds in $21 \mathrm{~cm}$ surveys have an optical counterpart, clouds with masses greater than $10^{8} \mathrm{M}_{\odot}$ would be detected in our statistics analysis, with reasonable assumptions regarding their $\mathrm{M}(\mathrm{HI}) / \mathrm{L}_{B}$ ratios. The main result of Telles \& Maddox can be summarized in Figure 1. The projected cross-correlation measurements are marginally lower than the predictions expected for a sample of normally clustered galaxies, and lie between the auto-correlation functions of normal galaxies and HII galaxies. The lower amplitude emphasizes, that there is not a large excess of near neighbors around the HII galaxies compared to normal galaxies. Again 
this is incompatible with suggestion that HII galaxies are triggered by tidal interactions with nearby low-mass galaxies.

These results put further limits on the properties of the possible low mass candidate triggers of star formation in HII galaxies. These may be low mass companions at distances lower than about one or two hundred pc, otherwise we would have picked them up in the correlation function analysis. Pustilnik et al. (2001), in another statistical analysis of the environment of 86 low mass galaxies with active star formation from the Second Byurakan Survey, find indication for close companions that might have been missed out in our cross-correlation function. In any case, it has become clear that some (rather large) fraction of HII galaxies are indeed isolated.

The point here is not only the presence of candidate triggers but the mechanism of star formation triggering. It has been shown in past studies that star formation is enhanced by galaxy interactions, with clear evidence in large mergers, as in the high luminosity, high mass end of the starburst phenomena (e.g. the ultra-luminous IRAS galaxies); and other examples of strong tidal effects. The fact that HII galaxies are dominated by a large emission line region has inspired the hypothesis that one has to have a very strong tidal agent to make all the gas rapidly pile up in the center of the galaxy so that the burst would be seen as one large event. The observations of structures in the ISM and the presence of different knots of SF, may free us from the idea of a large single event but indicate that SF may have formed locally and propagated. For this a weak tidal force may suffice, such as the mechanism proposed by Icke (1985). Shocks, due to internal or external agents, causing supersonic motions to propagate, producing high pressures and high density clouds enable the formation of the stellar clusters. Once they are formed their evolution may regulate further propagation of star formation. Evidence for the presence of stellar clusters in starbursts is growing and one may ask whether they are the cause or the effect of the starburst and in what conditions it may be one case or the other.

\section{Structure and Supersonic Motions in HII Galaxies}

Our high resolution echelle observations of a sample of HII galaxies (Telles, Múnoz-Túñon \& Tenorio-Tagle 2001) showed a strong variation in the line profiles across the emitting region and even in the most compact sources, there is an indication of separate knots of star formation (SF) evolving concurrently within the galaxy nucleus. The presence of multiple knots of star formation within the line emitting regions is also observed on high spatial resolution images, in particular in the near-IR, where we also identified knots which are possible super stellar clusters (SSC).

The structure found in H II galaxies has profound implications on several topics. In particular on issues such as star formation and its possible sequential propagation in H II galaxies, and how the ISM is structured in these galaxies. Another issue central in this field of research is the validity of the interpretation, and use of the empirical correlations, of size and luminosity $v s$ their supersonic line widths for high redshift galaxies. These correlations were first found for Giant H II Regions by Terlevich \& Melnick (1981) and Melnick et al. (1987) and extended to H II galaxies by Melnick et al. (1988) and Telles \& Terlevich (1993). 

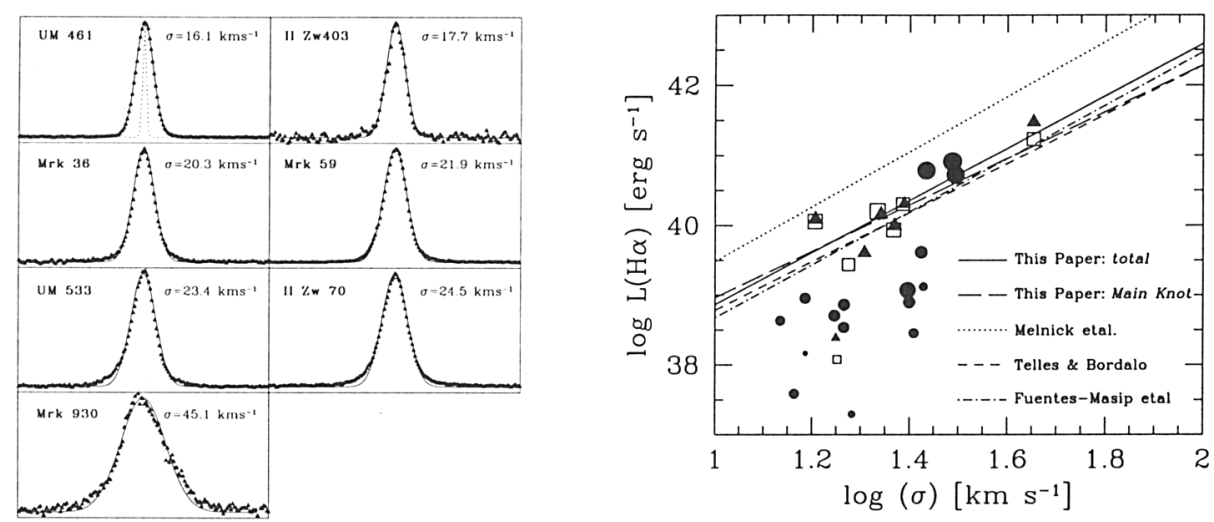

Figure 2. a. Emission line profiles of HII galaxies on the $\mathrm{H} \alpha$ line. Also shown are the Gaussian fits used to derive the final gas velocity widths. $b$. The luminosity - line width relation. Points: total apertures (solid triangles), Main Knot (open squares), other regions or knots (solid circles). Point sizes represent relative $\mathrm{H} \alpha$ surface brightness. The lines are simple linear square fits for our data and the statistical works from the literature.

The similarity of these relations with the Faber-Jackson relation for elliptical galaxies and the Tully-Fisher for late type spirals have led these investigators to propose that gravity is the dominant mechanism ruling the overall dynamics of HII galaxies. Other competing theories invoke stellar evolution with massive star winds and supernovae to be responsible for the inner motions in HII galaxies. However, the fact that line profiles are observed to be well fitted by gaussians (Figure 2a.) indicate that a continuous source of energy input is required. In addition, the [L-R- $\sigma$ ] correlations (Figure $2 \mathrm{~b}$ ). find a natural explanation in the Virial Theorem, which makes the gravity model the most appealing theory for the motions in HII galaxies.

Details of this study can be found in our paper (Telles et al. 2001) but our main points can be summarized: (i) our results confirm and extend the empirical correlations found for giant H II regions and H II galaxies. We indicate the possible effect of a second parameter in the relations similar to the fundamental plane for elliptical galaxies. (ii) enhanced spectral and spatial resolution seems to unveil an intricate structure in H II galaxies. (iii) H II galaxies when resolved, present several emitting knots with a variety of shapes, luminosity and $\sigma$ values. (iv) The intrinsic properties (luminosity, velocity dispersion) of a galaxy are dominated by the central (core) component.

A fine calibration of these relations for local $\mathrm{H}$ II galaxies may be of great importance if used as a distance indicator of galaxies at large redshift, since $\mathrm{H}$ II galaxies are easy to find at great distances (Melnick, Terlevich \& Terlevich 2000). 

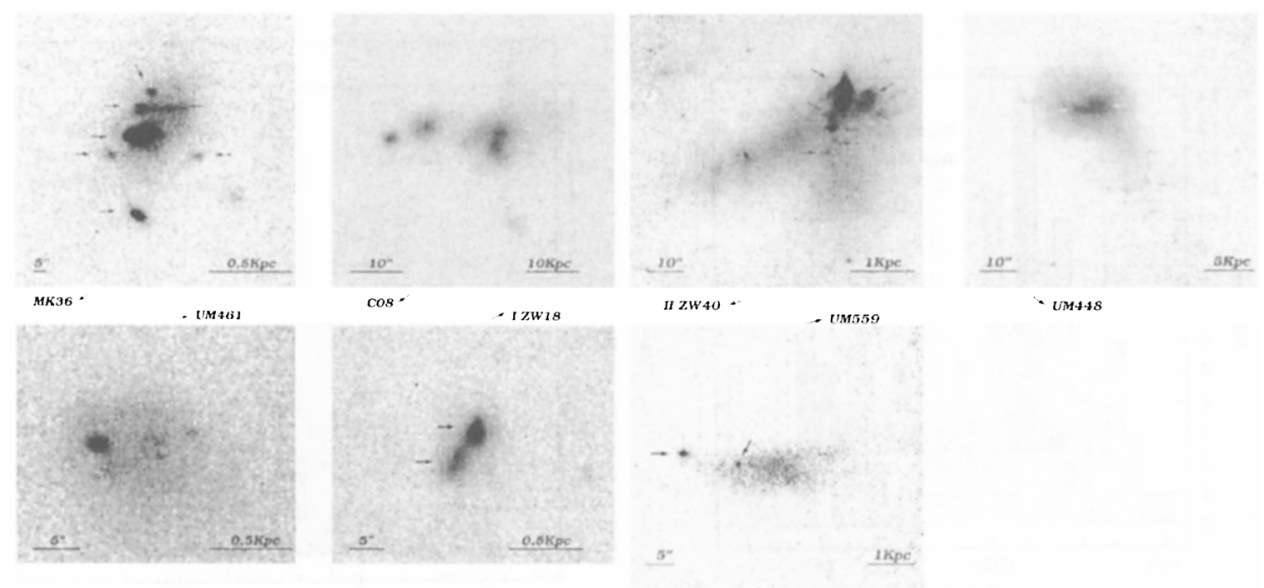

Figure 3. J band images of our UKIRT sample of HII galaxies. Arrows identify some of the SSC detected.

\section{The History of Star Formation through their Stellar Clusters}

Our near-IR images of $\mathrm{H}$ II galaxies have revealed supercluster-sized objects (SSC) within the star forming regions similar to those initially detected in HST ultraviolet images of starburst galaxies (Meurer et al. 1995; Vacca 1994, Ostlin et al. 1998). In many cases these knots form continuous structures, which suggests that star formation may have propagated across the starburst region. These SSCs appear to be a common feature in the starburst regions of merging galaxies (Surace \& Sanders 1999, see also Whitmore's and O'Connell's contribution), and have also been identified in a sample of Wolf-Rayet galaxies in ground based optical observations by Mendez \& Esteban (2000). Our present study, which will be appearing in a forthcoming paper in the near future (Telles, Sampson \& Tapia, 2001, in preparation), have shown that these SSC are more clearly identified in the near-infrared. Figure 3 shows $\mathrm{J}$ band images of our sample observed at UKIRT with the identification of some of the most visible clusters. We have attempted to age date the individual star clusters, thus having an alternative method of determining the star formation history of the galaxy. This seems to do a better job when comparing with models of population synthesis since we disentangle the need to assume a history of star formation a priori to age date the galaxy from its integrated light because the assumption of an instantenous burst for each individual stellar cluster is better justified.

We have used the Starburst99 model (Leitherer et al. 1999) of population synthesis to compare with our observations. Many words of caution must be given at this stage but I will defer these to the paper and only cite that one must worry about the choices of model parameters such as IMF, extinction, metallicity, nebular emission and dust emission. Due to the wide choices of model parameters and assumptions, it may not be possible or useful to derive the absolute ages from these comparisons, but the relative ages of the SSC from 

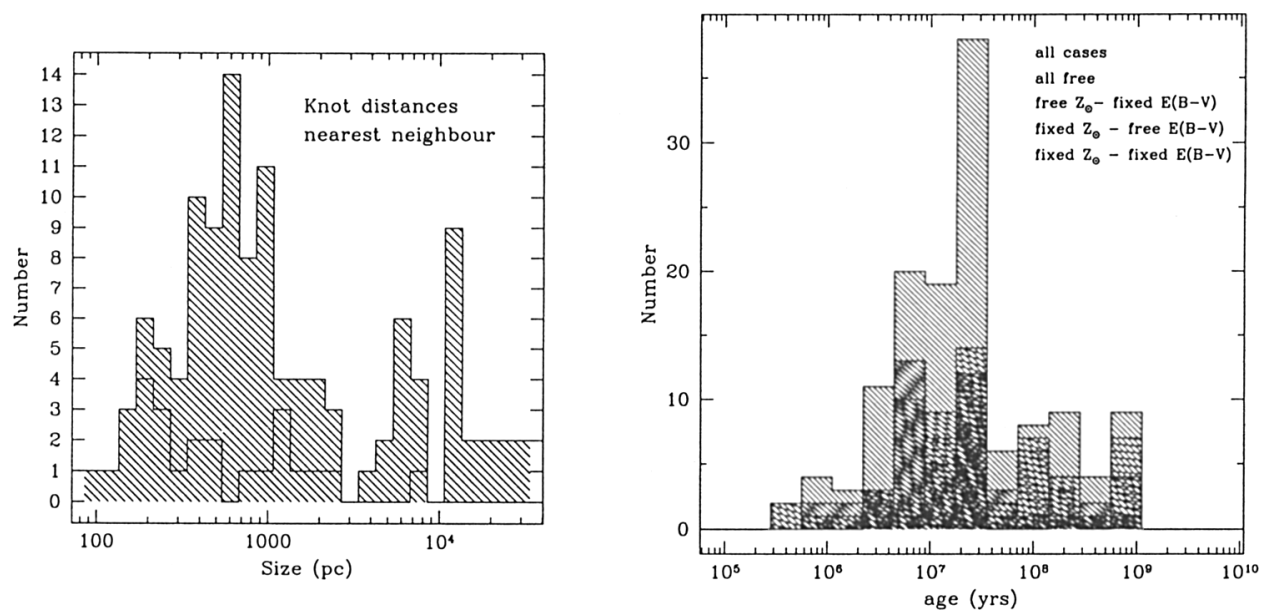

Figure 4. a. Distribution of distances from cluster to cluster. Inner histogram represents the nearest neighbors $\left(\mathrm{H}_{o}=65 \mathrm{~km} \mathrm{~s}^{-1} \mathrm{M}^{-}\right.$1.). b. Distribution of derived ages. Details are given in our paper. Only the overall results are intended to be noted here.

one single galaxy may be useful to describe the temporal and spatial patterns of Star Formation.

\subsection{Temporal and Spatial properties of Star Formation}

We have combined the information of our observations in the optical V R I and near-infrared J H K bands to compare with the model predictions. Our preferred choice of model metallicity is the one derived from spectroscopy and assumed to be an upper limit to the metallicity of the stellar population. Our preferred choice of extinction is also derived from spectroscopy measured by the Balmer decrement $(\mathrm{H} \alpha / \mathrm{H} \beta$ ratio) and assumed to be also an upper limit to the extinction in the continuum $\left(\mathrm{E}(\mathrm{B}-\mathrm{V})_{\text {cont }}=0.44 \times \mathrm{E}(\mathrm{B}-\mathrm{V})_{\text {gas }}\right)$ as given by Calzetti et al. (2000). Despite the preferred (fixed) values of metallicity or extinction, we ran the models of all metallicities and let the extinction also be a free parameter in order to verify which best represented all our combined observations. I will present here the overall results for all galaxies and super stellar cluster combined and refer the results for individual clusters and galaxies to the paper.

Figure 4a shows the histogram of all distances of a SSC to another in single galaxies for all galaxies. The inner histogram shows the nearest neighbors only. It can be seen that typically the nearest neighbor distance is about a few hundred pc. Figure $4 \mathrm{~b}$ shows a histogram of the derived ages of the individual clusters. I will not mention details of these analyses here but results seem to indicate typical ages of a few $10^{7}$ yrs.

Therefore we may speculate from this preliminary analysis that either SF propagates very rapidly $\left(<10^{8} \mathrm{yrs}\right)$ or it is synchronized in scales of hundreds of parsec so that a large burst can be observed to dominate the whole extent of 

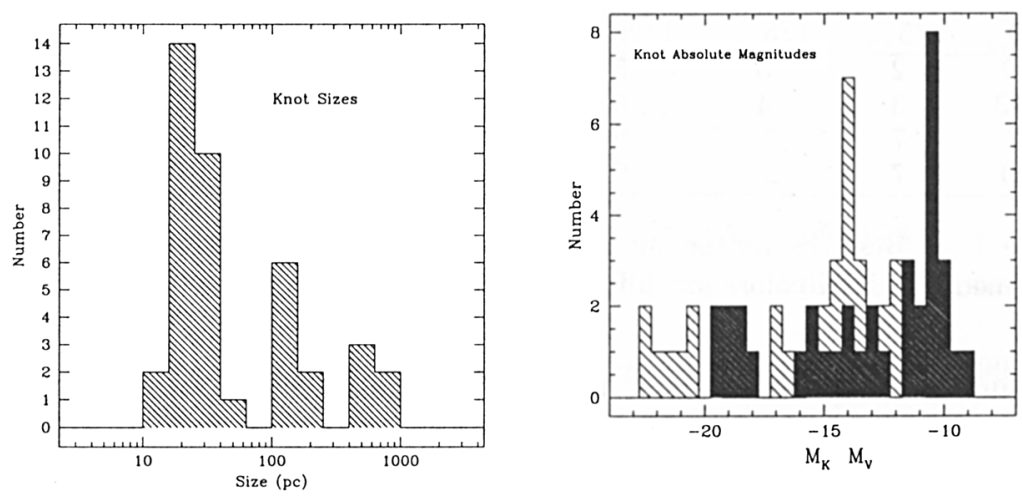

Figure 5. Distribution of Sizes and Absolute Magnitudes of SSCs.

an HII galaxy. Star Formation propagation has been proposed and studied since the late 70's (Gerola \& Seiden 1978; see also Nomura \& Kamaya 2001). We have also learned a great deal from the properties observed in the best laboratory for the study of massive star formation in a sub-solar metallicity environment, the giant HII region 30 Dor in the LMC. Grebel \& Chu (2000) show how SF may have propagated in this smaller analog to HII galaxies.

\subsection{Are these SSC similar to those observed in interacting galaxies?}

A first question one may pose is how these SSC in HII galaxies compare with those in strongly interacting or merging giant systems observed with the HST. Figure 5 shows the histograms of sizes and absolute magnitudes of all SSC. Disregarding the largest and most luminous ones which represent the nuclear regions, and are actually an ensemble of unresolved clusters, we find the SSC to have typical sizes of about $20 \mathrm{pc}, \mathrm{M}_{V} \approx-10$ and $\mathrm{M}_{K} \approx-14$. Assuming the results of the stellar models, these numbers represent a stellar cluster of one million stars $\left(1-100 \mathrm{M}_{\odot}\right)$ with a Salpeter IMF, not disimilar to the ones observed in other starbursts. We may have identified the elementary entities of which starbursts consist.

\subsection{Is there anything wrong with the comparison of models and observations of low metallicity galaxies?}

A first analysis of our overall results is summarized in Table 1 . The first 5 columns represent the number of SSC for which that particular metallicity has proven to be the best result. The last two columns tell you whether these results were obtained for a preferred chosen metallicity ( fixed) or left as a free parameter (free). Columns 6 and 7 give the corresponding mean age or extinction with their respective rms (not an error).

If we take these results at face value, despite all other complications, one can say that the models at low metallicity are not as red as they should be 


\begin{tabular}{ccccccccc}
\hline \multicolumn{1}{c}{ Starburst99 } & \multicolumn{1}{c}{} & & & & \\
\hline $\mathrm{a}$ & $\mathrm{b}$ & $\mathrm{c}$ & $\mathrm{d}$ & $\mathrm{e}$ & & & \\
\hline $2 \mathrm{Z}_{\odot}$ & $\mathrm{Z}_{\odot}$ & $2 / 5 \mathrm{Z}_{\odot}$ & $1 / 5 \mathrm{Z}_{\odot}$ & $1 / 20 \mathrm{Z}_{\odot}$ & $\langle\log (t)\rangle$ & $\langle\mathrm{E}(\mathrm{B}-\mathrm{V})\rangle$ & metal. & ext. \\
\hline 21 & 9 & 2 & 3 & 5 & $7.17 \pm 0.65$ & $0.24 \pm 0.25$ & free & free \\
14 & 13 & 3 & 4 & 6 & $7.30 \pm 0.57$ & $0.20 \pm 0.07$ & free & fixed \\
0 & 0 & 7 & 24 & 9 & $7.52 \pm 0.86$ & $0.37 \pm 0.26$ & fixed & free \\
0 & 0 & 7 & 24 & 9 & $7.64 \pm 0.76$ & $0.20 \pm 0.07$ & fixed & fixed \\
\hline
\end{tabular}

Table 1. Best results for our comparison of Starburst99 model with our combined VRIJHK colors for different metallicities and extinction

to reproduce the observed colors, because the best results are given by high metallicity models (model a or b), regardless of extinction (which is shown to vary within one galaxy), while we know that these are low metallicity systems. A K band excess has also been pointed out by Leo Vanzi (this conference) in his analysis of SBS0335-052. Therefore, these best results cannot be right.

The problem may be caused by a number of things: i) We are using the wrong models; we cannot for instance detect clusters with ages greater than 1 Gyr. We have tested this hypothesis by using Bruzual \& Charlot (2000) models but our present conclusions do not seem to change. ii) there may be an important contribution from dust emission. Although this has to be tested by observations at longer wavelengths, it is unlikely because emission line ratios do not point that way. iii) most likely, models at low metallicity are failing to reproduce the observations. One possible reason, among others, is that evolutionary tracks at low metallicity do not seem to reproduce either the observed numbers or colors of Red Supergiants (Maeder 2001, private communication). Stellar models which include rotation are being devised to produce a longer and more numerous RSG phase at low metallicity.

\section{Some Conclusions}

- Stellar Cluster formation and evolution is the mode of SF in HII galaxies, and is responsible for the magnitude and efficiency of the present burst.

- The properties of SSC in HII galaxies are similar to the SSC in interacting galaxies, and may be generic in a starburst.

- HII galaxies are not triggered by strong tidal agents. Weak tidal agents may be present at distances of only a few hundred parsec and masses of only $\approx 10^{8} \mathrm{M}_{\odot}$. A significant fraction may not be triggered at all and internal mechanism may be at play to let loose SF and its propagation.

Acknowledgments. I am deeply indebted to my collaborators Casiana Muñoz-Tunón, Guillermo Tenorio-Tagle, Jorge Melnick, Mauricio Tapia, Steve Maddox and Roberto Terlevich. I also thank my students Vinicius Bordalo and Leda Sampson for their hard work in dealing with the data and figures. Finally, I thank the organizers and the IAU for their financial support and for such a fruitful meeting. 


\section{References}

Calzetti, D. et al. , 2000, ApJ, 533, 682

Campos-Aguilar,A., Moles,M., Masegosa,J., 1993, AJ, 106, 1784

Cairós, L.M., Vilchez, J.M., González-Pérez, J.N., Iglesias-Páramo, J. and Caon, N. 2001, ApJS, 133, 321

Doublier, V., Comte, G., Petrosian, A., Surace, C, Turatto, M., 1997, A\&AS, 124,405

Gerola,H. \& Seiden,P.E., 1978, ApJ, 223, 129

Grebel,E.K. \& Chu, Y.-H, 2000, AJ,119,787

Icke, V., 1985, A\&A, 144,115

Leitherer et al. 1999, ApJS., 123, 3

Marlowe, A.T., Meurer, G.R. \& Heckman, T.M., 1999, ApJ, 522, 183

Melnick,J., 1987, in "Starburst and Galaxy Evolution", T.X.Thuan, T. Montmerle \& J.Tran Thanh Van eds., editions Frontières Gif Sur Yvette, France, p. 215

Melnick,J., Moles M., Terlevich R. \& Garcia-Pelayo J.M., 1987, MNRAS, 226, 849

Melnick,J., Terlevich R. \& Moles M., 1988, MNRAS, 235, 297

Melnick,J., Terlevich,R. \& Terlevich,E., 2000, MNRAS, 311, 629

Meurer, G. R., Heckman, T. M., Leitherer, C., Kinney, A., Robert, C., \& Garnett, D. R. 1995, AJ, 110, 2665

Mendez,D.I. \& Esteban, C., 2000, A\&A, 359:493

Nomura, H. \& Kamaya, H., 2001, ApJ, 1211024

Ostlin et al. 1998, A\&A, 335, 850

Ostlin, G. \& Kunth, D. 2000, A\&ARv, 10, 1

Pustilnik, S.A., Kniazev, A.Y., Lipovetsky, V.A. \& Ugryumov, A. A., 2001, astro-ph/0104334

Sargent W.L.W. \& Searle L., 1970, ApJ, 162, L155

Surace, J. A. \& Sanders, D. B., 1999, ApJ, 512, 162

Taylor,C.L 1997, ApJ, 480, 524

Telles, E. \& Maddox, s. 2000, MNRAS, 311, 307

Telles,E. \& Terlevich,R., 1993, Ap\&SS, 205, 49

Telles,E. \& Terlevich,R., 1995, MNRAS, 275, 1

Telles, E. \& Terlevich, R. 1997, MNRAS, 286, 183

Telles, E., Melnick,J. \& Terlevich,R. 1997, MNRAS, 288, 78

Telles,E., Muñoz-Tuñón, C. \& Tenorio-Tagle, G. 2001, ApJ, 548, 671

Terlevich, R. \& Melnick, J., 1981, MNRAS, 195, 839

Vacca, W.D., 1994, in "Violent Star Formation", ed Tenorio-Tagle, p 297

Vilchez,J.M., 1995, AJ, 110, 1090 


\section{Discussion}

H. Lamers: A warning for everybody who tries to model the energy distributions of clusters that contain a significant population of red supergiants. In the Geneva models of red supergiants the convection is artificially truncated to avoid numerical instabilities. This implies that the red supergiant models are too hot and not red enough. This effect may also plague the Starburst99 models.

L. Vanzi: You mentioned excess in the band observed in your sample of galaxies that you attribute to dust. But since the models do not work very well at low metallicity how can you be sure of that?

E. Telles: You cannot. The comparison is made with models that are not reproducing the colours we observe. In any case, apparently we may also be dealing with other competing effects, a dust emission contribution to the $\mathrm{K}$ light in the more luminous galaxies and emission line contribution in the lower luminosity galaxies where the dust content is smaller and the continuum is weaker. But it would be interesting to observe the low luminosity galaxies at longer wavelengths to determine their dust content.

D. Forbes: Have you used the so-called Q parameter to study HII galaxies?

E. Telles: Not yet. In our first analysis we left extinction as a free parameter. We wanted to check the effect of extinction by direct comparison of stellar models with the combination of our colours. Best fit extinction values were obtained for the centers which show consistency with the Balmer decrement derived from the spectroscopic data. Inconsistencies were found for other clusters that indicates that internal extinction may vary from cluster to cluster. 